\title{
Breastfeeding Initiation among Women with Chronic Hypertension
}

\section{Leandro Cordero, $M D^{1 *}$, Michael R Stenger, $M D^{1}$, Mark B Landon, MD and Craig A Nankervis, $M D^{1}$}

${ }^{1}$ Department of Pediatrics, College of Medicine, The Ohio State University, USA

${ }^{2}$ Department of Obstetrics and Gynecology, College of Medicine, The Ohio State University, USA

*Corresponding author: Leandro Cordero, MD, Professor Emeritus, Department of Pediatrics, The Ohio State University Wexner Medical Center, N118 Doan Hall, 410 W. $10^{\text {th }}$ Avenue, Columbus, Ohio, 432101228, USA, Tel: 614-293-8660, Fax: 614-293-7676

\begin{abstract}
Background: Chronic hypertension (CHTN) affects 1-1.5\% of all pregnant women and approximately one third require antihypertensive medications (meds) before pregnancy or during the first 20 weeks of gestation.
\end{abstract}

Objective: To determine breastfeeding (BF) initiation rates of women with $\mathrm{CHTN}$ and their association with pregestational body mass index (BMI $\left.\mathrm{kg} / \mathrm{m}^{2}\right)$.

Methods: Retrospective study of 111 women with CHTN requiring meds and 206 with $\mathrm{CHTN}$ not requiring medication (without meds) who delivered at $\geq 34$ weeks of gestation. Intention to BF exclusively or partially was declared prenatally. At discharge, exclusive $\mathrm{BF}$, partial BF and formula feeding (FF) were determined.

Results: CHTN-meds and without meds groups were similar in primiparity (41 \& 46\%), superimposed preeclampsia (23 \& 21\%), intention to exclusively BF (91 \& 87\%), vaginal delivery (56 \& 61\%), GA at delivery (38 \& 38 weeks), late preterm (16 \& 14\%) and admission to NICU (17 \& 16\%). These groups differed in maternal age (32 \& 30y) and black race $(22 \& 34 \%)$. At discharge, exclusive BF (50 \& 47\%), partial BF (32 \& 35\%), FF (18 \& 17\%) and BF initiation (82 $\& 82 \%$ ) were similar among groups. Comparison between 130 women with BMI 30-39 (obese) and 146 with BMI $\geq$ 40 (morbidly/extremely obese) showed vaginal delivery to be less common $(67 \& 47 \%)$ and primary cesarean to be more frequent (17 \& $32 \%)$ among the latter group. At discharge, both groups were comparable in exclusive BF (48 \& 47\%), partial BF (34 \& 35\%), FF (18 \& 18\%) and BF initiation rate $(82 \& 82 \%)$.

Conclusion: BF initiation rates for women with $\mathrm{CHTN}$ meds and CHTN without meds were similar. Morbid obesity did not negatively affect BF initiation rates.

\section{Background}

Chronic hypertension (CHTN) affects $1-1.5 \%$ of all pregnant women and may lead to adverse maternal and neonatal outcomes [1-3]. The prevalence of CHTN in pregnancy has increased more than ten-fold in the US since 1970 and coincides with an increase in obesity, type 2 diabetes and advanced maternal age [4-6]. Due to severity of illness, one third of women with CHTN require antihypertensive medications (meds) before the onset of pregnancy or starting during the first 20 weeks of gestation [1-3,7]. According to current guidelines, antihypertensive medications should be reserved for women with severe hypertension or with specific renal or cardiovascular co-morbidities [1-3]. CHTN is associated with obesity, superimposed preeclampsia, cesarean delivery, cardiovascular disease, placental abruption, fetal growth restriction and maternal and perinatal death $[1,3,8,9]$. Adverse neonatal outcomes include indicated preterm delivery, cesarean birth, poor fetal growth, preeclampsia and neonatal intensive care unit (NICU) admission $[3,8,9]$.

Hypertensive disorders during pregnancy, particularly those complicated by preeclampsia, have long-term and possibly permanent consequences for mothers and their infants $[10,11]$. Recent guidelines encourage $\mathrm{BF}$ among women with CHTN because most commonly used antihypertensive medications are found in low concentrations in breast milk $[1,2,3,12]$. Lactation has been recognized to provide short- and long-term health benefits to mothers and their infants following healthy pregnancies as well as those compromised by different

Citation: Cordero L, Stenger MR, Landon MB, Nankervis CA (2021) Breastfeeding Initiation among Women with Chronic Hypertension. Int J Womens Health Wellness 7:118. doi.org/10.23937/24741353/1510118

Accepted: January 28, 2021: Published: January 30, 2021

Copyright: (C) 2021 Cordero L, et al. This is an open-access article distributed under the terms of the Creative Commons Attribution License, which permits unrestricted use, distribution, and reproduction in any medium, provided the original author and source are credited. 
co-morbidities [3,13-16]. In spite of the above, information on BF initiation among women with CHTN remains scarce.

\section{Objective}

To compare BF initiation among women with CHTNmeds and CHTN without meds. A secondary objective was to compare $\mathrm{BF}$ initiation rates among subclasses of obese women with CHTN.

\section{Subjects and Methods}

This retrospective cohort investigation was approved by the Institutional Review Board at The Ohio State University Wexner Medical Center. Electronic maternal and neonatal records (2016-18) were reviewed. CHTN was defined as hypertension diagnosed before conception or at $<20$ weeks of gestation in the current pregnancy according to established clinical and laboratory criteria [1-3]. Indications for treatment of CHTN with medication were in accordance with established ACOG guidelines [1-3]. Women were categorized by body mass index (BMI) as normal $\left(18-24.9 \mathrm{~kg} / \mathrm{m}^{2}\right)$, over weight $(25-29.9$ $\left.\mathrm{kg} / \mathrm{m}^{2}\right)$, obese $\left(30-39.9 \mathrm{~kg} / \mathrm{m}^{2}\right)$, morbidly obese (40-49.9 $\left.\mathrm{kg} / \mathrm{m}^{2}\right)$ and extremely obese $\left(\geq 50 \mathrm{~kg} / \mathrm{m}^{2}\right)$.

The study population consisted of women with CHTN who intended to BF (exclusively or partially) at discharge and their singleton infants delivered at $\geq 34$ weeks gestation. Pregnancies affected by diabetes mellitus or major fetal malformations were not included. Upon arrival to labor and delivery, each woman described her past $\mathrm{BF}$ experience and her intention to BF. Our family-centered care system has rooming-in available and full-time lactation consultants whose services are offered to all women regardless of their infant feeding preference.

Per our hospital practice, any symptomatic infants were directly transferred from the delivery room to the NICU for further care. If the condition of the mother and her infant following delivery allowed, maternal-infant interactions such as holding, skin-to-skin contact, and BF were encouraged. Asymptomatic infants able to feed were transferred to the newborn nursery for routine care and glucose monitoring if applicable. Delivery room and postpartum maternal-infant interactions were observed and documented by the obstetrical, newborn nursery and NICU nursing staffs and by lactation consultants.

Screening for hypoglycemia (blood glucose $<40 \mathrm{mg}$ / dl) was done via serial point of care testing (Accu-Chek ${ }^{\circ}$ ) or by plasma glucose measurement in the laboratory (Beckman Coulter AU5800, Beckman Coulter Inc., Brea, CA, U.S.A.) starting within the first hour of life after the first feeding and every 2-4 hours thereafter as needed. Asymptomatic infants in the newborn nursery with hypoglycemia were promptly BF or formula fed (FF) and those with recurrent hypoglycemia were treated with intravenous (IV) dextrose. On admission to the NICU, most infants were started on IV dextrose and those who were able to feed were BF or FF.

BF was considered initiated if, during the 24 hours preceding hospital discharge, infants were BF exclusively or BF partially. Exclusive BF was defined by direct feedings from the breast, by expressed breast milk (EBM) alone or in combination with direct BF or by donor human milk. Partial BF was defined by direct $B F$ or EBM in combination with FF. Due to the retrospective study design, no follow-up information was available on infant feeding practices after hospital discharge.

\section{Statistical Analysis}

Comparisons between women with CHTN-meds and CHTN without meds were made with two-sample t-tests for continuous variables and Chi square tests for categorical variables. Significance was established at a $p$ value $<0.05$. A secondary analysis was designed to ascertain clinical and demographic characteristics and BF outcomes of 130 obese (BMI $30-39.9 \mathrm{~kg} / \mathrm{m}^{2}$ ) and 146 morbidly and extremely obese $\left(\mathrm{BMI} \geq 40 \mathrm{~kg} / \mathrm{m}^{2}\right)$ women with CHTN.

\section{Results}

The study population consisted of 317 women with CHTN (111 CHTN-meds and 206 CHTN without meds).

\section{Comparison of women with CHTN-meds and CHTN without meds}

Clinical and demographic characteristics of women with CHTN-meds and CHTN without meds are shown in Table 1. Most variables were similar between the groups although mother's age was higher and the percent of black women were lower in the CHTN-meds group. Consistent with the diagnosis and treatment of women in the CHTN-meds group, the most common medications prescribed were labetalol $74(67 \%)$, nifedipine $9(8 \%)$, amlodipine $9(8 \%)$, metoprolol $9(8 \%)$, methyldopa 8 (7\%) and carvedilol 2 (2\%). At discharge from the hospital, $95 \%$ of women from the CHTN-meds and $15 \%$ of those in the CHTN without meds groups received oral antihypertensive medications.

CHTN with superimposed preeclampsia affected $23 \%$ women with CHTN-meds and $21 \%$ with CHTN without meds. All women with CHTN with superimposed preeclampsia with severe features received 24 hours of postpartum intravenous magnesium sulfate for seizure prophylaxis.

Smoking during pregnancy ( 6 \& 10\%) was similar between the CHTN-meds and CHTN without meds groups. However, history of smoking (22 \& 13\%) was more common among the CHTN without meds group. The rates of vaginal deliveries (56\& 61\%), primary cesarean (23 \& $23 \%$ ) and repeat cesarean ( 21 \& 16\%) were similar between groups. 
Table 1: Comparison of women with CHTN-meds and CHTN without meds.

\begin{tabular}{|c|c|c|c|}
\hline & With Medication & Without Medication & $p$ \\
\hline Mother-Infant dyads no. & 111 & 206 & \\
\hline CHTN no. (\%) & $86(77)$ & $162(79)$ & NS \\
\hline CHTN with superimposed preeclampsia no. (\%) & $25(23)$ & $44(21)$ & NS \\
\hline Mothers age $(\mathrm{y})$ mean $\pm \mathrm{SD}$ & $32 \pm 6$ & $30 \pm 6$ & 0.01 \\
\hline \multicolumn{4}{|l|}{ Race } \\
\hline Black no. (\%) & $25(23)$ & $70(34)$ & 0.04 \\
\hline White no. (\%) & $72(65)$ & $117(57)$ & NS \\
\hline Hispanic no. (\%) & $7(6)$ & $8(4)$ & NS \\
\hline Other no. (\%) & $7(6)$ & $11(5)$ & NS \\
\hline Smoking no. (\%) & $7(6)$ & $21(10)$ & NS \\
\hline Former smokers no. (\%) & $14(13)$ & $45(22)$ & 0.04 \\
\hline Body Mass Index $\mathrm{kg} / \mathrm{m}^{2}$ mean $\pm \mathrm{SD}$ & $38 \pm 9$ & $40 \pm 9$ & NS \\
\hline Body Mass Index kg/m² $\geq 35$ no. (\%) & $70(63)$ & $140(68)$ & NS \\
\hline Primiparous no. (\%) & $45(41)$ & $94(46)$ & NS \\
\hline \multicolumn{4}{|l|}{ Mode of Delivery } \\
\hline Vaginal no. (\%) & $62(56)$ & $125(61)$ & NS \\
\hline Primary cesarean no. (\%) & $25(23)$ & $47(23)$ & NS \\
\hline Repeat cesarean no. (\%) & $24(21)$ & $34(16)$ & NS \\
\hline Gestational age $(w)$ mean \pm SD & $38 \pm 2$ & $38 \pm 2$ & NS \\
\hline Preterm no. (\%) & $18(16)$ & $28(14)$ & NS \\
\hline Term no. (\%) & $93(84)$ & $178(86)$ & NS \\
\hline Mother length of stay $(d)$ mean \pm SD & $4 \pm 2$ & $4 \pm 3$ & NS \\
\hline
\end{tabular}

CHTN - Chronic hypertension

Table 2: Neonatal outcomes of infants born to women with CHTN-meds and CHTN without meds.

\begin{tabular}{|l|c|c|}
\hline & With Medication & Without Medication \\
\hline Mother-Infant Dyads no. & 111 & 206 \\
\hline Gender (males) no. (\%) & $62(55)$ & $112(54)$ \\
\hline Birthweight (g) mean \pm SD & $3062 \pm 603$ & $3192 \pm 533$ \\
\hline Gestational age (w) mean \pm SD & $38 \pm 2$ & $38 \pm 2$ \\
\hline All preterm no. (\%) & $18(16)$ & $28(14)$ \\
\hline Full-term no. (\%) & $93(84)$ & $178(86)$ \\
\hline Intrauterine Growth & & NS \\
\hline \multicolumn{1}{|c|}{ Appropriate for gestational age no. (\%) } & $89(80)$ & $162(79)$ \\
\hline \multicolumn{1}{|c|}{ Large for gestational age no. (\%) } & $11(10)$ & $22(11)$ \\
\hline Small for gestational age no. (\%) & $11(10)$ & $22(11)$ \\
\hline Admission to NICU no. (\%) & $18(16)$ & $33(16)$ \\
\hline Neonatal hypoglycemia no. (\%) & $14(13)$ & $28(14)$ \\
\hline Infant length of stay (d) mean \pm SD & $3 \pm 2$ & NS \\
\hline Discharged home with mother no. (\%) & $101(90)$ & NS \\
\hline
\end{tabular}

Neonatal outcomes of infants born to women with $\mathrm{CHTN}$-meds and $\mathrm{CHTN}$ without meds

Neonatal outcomes of infants born to women with CHTN-meds and CHTN without meds are shown in Table 2. Outcomes between the groups were comparable in the incidence of late prematurity (16 \& $14 \%)$, admission to NICU (16 \& 16\%) and infant length of stay $(3 \pm 2 \& 4$ $\pm 4 d$ ). Considering the similarities in diagnoses, 18 in- fants from the CHTN-meds group and 33 infants from the CHTN without meds group admitted to the NICU were combined for analysis. Of these 51 infants, 27 (53\%) were full-term and 24 (47\%) were late preterm. NICU admission diagnoses included respiratory distress (29\%), temperature instability-hypotonia-poor feeding (33\%), apnea-bradycardia-cyanosis (20\%), hypoglycemia $(10 \%)$, neonatal abstinence syndrome (4\%) and miscel- 
Table 3: BF at discharge for women with $\mathrm{CHTN}-$ meds and $\mathrm{CHTN}$ without meds.

\begin{tabular}{|c|c|c|c|}
\hline & With Medication & Without Medication & $p$ \\
\hline Mother-Infant Dyads no. & 111 & 206 & \\
\hline Prior Breastfeeding no.(\%) & $46(70)$ & $87(78)$ & NS \\
\hline \multicolumn{4}{|l|}{ Infant Feeding at Discharge } \\
\hline Exclusive breastfeeding total no. (\%) & $56(50)$ & $97(47)$ & NS \\
\hline Direct breastfeeding BF no. (\%) & $43(77)$ & $80(82)$ & NS \\
\hline Expressed breast milk no. (\%) & $13(23)$ & $18(19)$ & NS \\
\hline Partial breastfeeding total no. (\%) & $35(32)$ & $72(35)$ & NS \\
\hline Direct breastfeeding and Formula no. (\%) & $22(63)$ & $45(63)$ & NS \\
\hline Expressed breast milk and Formula no. (\%) & $13(37)$ & $27(38)$ & NS \\
\hline Formula feeding no. (\%) & $20(18)$ & $36(17)$ & NS \\
\hline Breastfeeding Initiation no. (\%) & $91(83)$ & $169(82)$ & NS \\
\hline
\end{tabular}

laneous (4\%). Fourteen of the 51 infants (27\%) stayed in the NICU for less than one day and seven (14\%) infants stayed two days. Among the 317 infants of the CHTN groups combined, there were $33(10 \%)$ small for gestational age (SGA), of them only seven were admitted to the NICU. All 317 mothers and their infants were discharged home in good condition.

\section{Prior BF experience and early BF among women with CHTN-with and CHTN without meds}

The percent of multiparous women with CHTN in either group were similar ( $59 \& 54 \%$ ). Relevant to our study, prior BF experience among them was also comparable (70 \& 78\%). Intention to exclusively BF was similar among women with CHTN-meds (85\%) and women with CHTN without meds (85\%).

Immediate transfer of symptomatic infants to the NICU was required by $13 \%$ of the CHTN-meds and $12 \%$ of the CHTN without meds group. During the first two postpartum hours the following mother-infant interactions were recorded: BF (68 \& 67\%) and skin-to-skin contact without $\mathrm{BF}$ for the remaining dyads. At the end of the hospital stay, ( $82 \& 82 \%$ ) women of either CHTN group had BF at least once.

Further analysis of mode of delivery on early BF showed that among 187 women from both groups combined who delivered vaginally, $142(76 \%)$ were able to BF within two hours of birth while only 72 of 130 (55\%) of infants delivered by cesarean were able to BF within two hours of birth ( $p$ 0.0002). Over time, however, these differences did not persist since at discharge, 153 of the 187 (82\%) infants born vaginally and 108 of 130 (83\%) of those born by cesarean had initiated BF. Of note, at discharge, exclusive BF was also similar for women who delivered vaginally and for those who delivered by cesarean (50 \& 47\%).

\section{BF at discharge for women with $\mathrm{CHTN}-m e d s$ and CHTN without meds}

At the time of discharge the rates of exclusive, par- tial $\mathrm{BF}, \mathrm{FF}$ and $\mathrm{BF}$ initiation were similar between the two groups (Table 3). Four of the 13 dyads in the CHTNmeds group and 6 of the $18 \mathrm{CHTN}$ without meds group received exclusive EBM while in the remaining instances, EBM complemented direct BF. Nine of the $56(16 \%)$ infants in the CHTN-meds group and 17 out of $97(18 \%)$ in the CHTN without meds group that BF exclusively at discharge received formula supplementation during their hospital stay. Two infants in the CHTN-meds group and four in the CHTN without meds group received donor human milk during their hospitalization. Lactation consults were given to all women in either group with the median number of consultations per dyad being 2 (range 1-6).

\section{Breastfeeding initiation among women with CHTN according to pregestational BMI}

The entire population of 317 women was divided into groups according to pregestational BMI as follows: 5 (2\%) normal, 36 (11\%) overweight, $130(41 \%)$ obese, 101 (32\%) morbidly obese and 45 (14\%) extremely obese. Comparisons of clinical and demographic characteristics of 130 obese and 146 morbidly/extremely obese women combined are shown in Table 4. CHTNmeds occurred with similar frequency in both groups (38 \& 32\%). The incidence of superimposed preeclampsia was also similar (23 \& 23\%). Other comparable characteristics were primiparity (39 \& 48\%), mothers age (31 \& 31y) as well as advanced maternal age ( 23 \& 20\%). Vaginal delivery was less common (47 \& 67\%) and primary cesarean (17 \& $32 \%$ ) was more common among women in the morbidly/extremely obese BMI group. At discharge, both groups were comparable in exclusive $B F$ (48 \& 47\%), partial BF (34 \& 35\%), FF (18 \& 18\%) and BF initiation rate ( $82 \& 82 \%)$.

\section{Discussion}

CHTN is a common medical complication of pregnancy and approximately one third of women require antihypertensive medications before conception or during the first 20 weeks of gestation and beyond [13]. The rate of CHTN during pregnancy across the world 
Table 4: Breastfeeding initiation among women with $\mathrm{CHTN}$ according to pregestational Body Mass Index.

\begin{tabular}{|c|c|c|c|}
\hline & $\begin{array}{c}\mathrm{BMI} \mathrm{kg} / \mathrm{m}^{2} \\
30-39\end{array}$ & $\begin{array}{c}\text { BMI } \mathrm{kg} / \mathrm{m}^{2} \\
\geq 40\end{array}$ & $p$ \\
\hline Mother-Infant Dyads no. & 130 & 146 & \\
\hline CHTN no. (\%) & $100(77)$ & $113(77)$ & NS \\
\hline $\mathrm{CHTN}$ with medications no. (\%) & $49(38)$ & $47(32)$ & NS \\
\hline CHTN with superimposed preeclampsia no. (\%) & $30(23)$ & $33(23)$ & NS \\
\hline Mothers age $(y)$ mean $\pm S D$ & $31 \pm 6$ & $31 \pm 6$ & NS \\
\hline \multicolumn{4}{|l|}{ Race } \\
\hline Black no. (\%) & $34(26)$ & $48(33)$ & NS \\
\hline White no. (\%) & $80(62)$ & $85(58)$ & NS \\
\hline Other no. $(\%)$ & $16(12)$ & $13(9)$ & NS \\
\hline Primiparous no. (\%) & $51(39)$ & $71(48)$ & NS \\
\hline \multicolumn{4}{|l|}{ Mode of Delivery } \\
\hline Vaginal no. (\%) & $87(67)$ & $69(47)$ & 0.001 \\
\hline Primary cesarean no. (\%) & $22(17)$ & $47(32)$ & 0.004 \\
\hline Repeat cesarean no. (\%) & $21(16)$ & $30(21)$ & NS \\
\hline Mother length of stay $(d)$ mean \pm SD & $3 \pm 1$ & $4 \pm 4$ & NS \\
\hline Gestational age $(w)$ mean \pm SD & $38 \pm 1$ & $38 \pm 2$ & NS \\
\hline Preterm no. (\%) & $17(13)$ & $22(15)$ & NS \\
\hline Term no. (\%) & $113(87)$ & $124(85)$ & NS \\
\hline Birthweight $(\mathrm{g})$ mean \pm SD & $3129 \pm 538$ & $3247 \pm 581$ & NS \\
\hline Small for gestational age no. (\%) & $14(11)$ & $11(8)$ & NS \\
\hline Large for gestational age no. (\%) & $8(6)$ & $24(16)$ & 0.008 \\
\hline Admission to NICU no. (\%) & $17(13)$ & $28(19)$ & NS \\
\hline Infant length of stay $(d)$ mean $\pm S D$ & $3 \pm 3$ & $4 \pm 3$ & NS \\
\hline \multicolumn{4}{|l|}{ Infant Feeding at Discharge } \\
\hline Exclusive breastfeeding total no. (\%) & $62(48)$ & $69(47)$ & NS \\
\hline Direct breastfeeding no. (\%) & $49(79)$ & $53(77)$ & NS \\
\hline Expressed breast milk no. (\%) & $13(21)$ & $16(23)$ & NS \\
\hline Partial breastfeeding total no. (\%) & $45(34)$ & $51(35)$ & NS \\
\hline Direct breastfeeding and Formula no. (\%) & $32(71)$ & $28(55)$ & NS \\
\hline Expressed breast milk and Formula no. (\%) & $13(29)$ & $23(45)$ & NS \\
\hline Formula feeding no. (\%) & $23(18)$ & $26(18)$ & NS \\
\hline Breastfeeding initiation no. (\%) & $107(82)$ & $120(82)$ & NS \\
\hline
\end{tabular}

CHTN - Chronic hypertension

continues to rise in parallel to the obesity epidemic and increasing maternal age $[1,5,6,17]$. The severity of hypertension among women with CHTN-meds in our study seems validated by their almost universal need for antihypertensive treatment before, during and beyond discharge from the hospital $[1,2,7,12]$.

Rates of preeclampsia, cesarean delivery, preterm birth, admission to the NICU and disorders of fetal growth noted here are comparable to that reported by others $[8,9,18]$. The similarities of the maternal and neonatal outcomes in this investigation may be suggestive of the benefits of antihypertensive treatment of women with more severe CHTN, however, additional randomized trials are needed to determine the bene- fit of antihypertensive therapy in women with moderate hypertension in pregnancy $[1,2,7]$. Earlier studies have shown that in some instances, antihypertensive treatment may reduce the risk of severe hypertension without visible changes in the rates of adverse perinatal outcomes $[3,7]$. The incidence of preeclampsia reported here $23 \%$ for CHTN-meds and $21 \%$ for those in the CHTN without meds group is comparable to that reported by others $[8,11,19]$. Not surprising, the incidence of cesarean delivery increases in relation to the severity of superimposed preeclampsia on CHTN. In our study, primary cesarean rate among 248 women with CHTN was $20 \%$, whereas the rate among 69 CHTN with superimposed preeclampsia was $34 \%$. 
Short term benefits of BF among infants and children of healthy women or those challenged by high risk obstetrical conditions have been documented [19-23]. There is growing evidence that lactation has short- and long-term cardiovascular health benefits for women $[13,15]$. An early study of duration of BF on the incidence of maternal hypertension showed that women who never BF had an increased incidence of hypertension in later years compared to those who BF six months or longer [13]. Recently, a systematic review of four cross-sectional studies reported that history of lactation lowered the odds of hypertension among women of middle to old age [15]. Hypertensive disorders during pregnancy, in particular preeclampsia, may have long term and possibly permanent consequences for motor development in the offspring $[22,23]$.

Intention to $\mathrm{BF}$ is one of the strongest predictors of $\mathrm{BF}$ initiation in healthy as well as high risk obstetrical populations $[20,21,24]$. Despite the heterogeneity of morbidities and co-morbidities associated with high risk obstetrical pregnancies including CHTN, intention to BF remains comparable to that of the general population [25]. Unfortunately, a discordance exists among women with complex pregnancies between their intention to $\mathrm{BF}$ and their actual rate of $\mathrm{BF}$ initiation at the time of discharge $[20,21,24]$. There are many recognizable obstacles to BF initiation including lack of intention to BF, gestational and pregestational diabetes, preeclampsia, maternal obesity, cesarean delivery, premature birth, mother-infant separation, admission to the NICU and infant morbidities that lead to lower BF initiation rates $[16,20,21,24,26]$.

Our finding that women with CHTN-meds and CHTN without meds who intended to BF had a BF initiation rate of over $80 \%$ (exclusive or partial $\mathrm{BF}$ ) at discharge is significant because it compares favorably with that of the general healthy maternal population [25]. However, this success is tempered by the fact that only half of women from either CHTN group were able to exclusively $\mathrm{BF}$ their infants at the time of discharge well below the mandate of a national regulatory agency [27].

Both the American Academy of Pediatrics and the Academy of Breastfeeding Medicine recommend exclusive $\mathrm{BF}$ for all healthy infants during birth hospitalization and beyond [28-30]. However, these organizations acknowledge that other nutritional options may be needed to temporarily replace or supplement BF under well-defined circumstances (i.e., maternal and neonatal illness, late preterm infants). Healthcare providers must be aware that delays associated with some morbidities will further the need for alternatives to exclusive direct $\mathrm{BF}$ [28-30]. Due to their clinical condition or to the separation from their sick or prematurely born infants, not all women with CHTN-meds or CHTN without meds are able to BF shortly after birth. In that case, our practice is to provide mother's milk if obtained antenatally, EBM if tolerated, donor human milk if feasible or infant formula if prescribed by a physician $[27,29]$.

It is well known that milk expression by hand or with an electrical pump may help mothers overcome obstacles to early BF and therefore increase BF duration [31-35]. In line with current literature, it is hoped that women who direct BF with or without EBM at discharge would continue to provide exclusive or partial $B F$. On the other hand, feeding exclusively by EBM is concerning since available reports are pessimistic about BF duration following hospital discharge [34,35]. Several investigators concurred that exclusive EBM feeding should be recommended for full-term and premature infants only when medically necessary and not as a substitute for feeding directly from the breast $[33,34]$.

Obese women, even those without CHTN, are more likely to have complicated labors, cesarean deliveries and separation from their infants postpartum $[21,26,36]$. It has been reported that obese women have less intention to $\mathrm{BF}$, lower rates of $\mathrm{BF}$ initiation and shorter duration of exclusive and any BF $[16,36]$. Several years ago, in a study of 360 non-diabetic obese women who delivered macrosomic infants, we reported that intention to BF was similar for obese/very obese ( 75 \& $73 \%$ ) but it was lower (61\%) for morbidly obese and that $\mathrm{BF}$ initiation was $71 \%, 66 \%$ and $53 \%$, respectively [36]. We later realized that $\mathrm{BF}$ initiation rates were so low because like others, we included in our study populations both women who intended to $\mathrm{BF}$ as well as those who intended not to BF. Considering that women who do not intend to BF seldom change their mind and will exclusively FF their infants, in this investigation we included only women who intended to BF exclusively or partially $[21,24,36]$. As a consequence, the data presented here showed that BF initiation was higher among women who intended to $\mathrm{BF}$ and highlighted the obstacles that prevented some of them from achieving their desired BF goal.

In normal as well as in high risk pregnancies certain hospital practices may delay infant feeding (i.e., cesarean delivery, eye prophylaxis, vitamin $\mathrm{K}$ administration, blood glucose monitoring). While some of these practices may be postponed, others may be unavoidable especially in women with CHTN-meds and CHTN without meds who delivered prematurely. Cesarean delivery, a traditionally recognized obstacle to early mother infant interactions, occurs frequently among women with CHTN-meds and CHTN without meds $[1,2,8,26,37,38]$. Earlier, we reported that cesarean delivery increased from $38 \%$ among obese to $57 \%$ among those extremely obese [36]. In the present study, we observed 33\% cesarean deliveries among 130 obese/very obese and 53\% cesarean deliveries among 146 morbidly/extremely obese women with CHTN.

Cesarean delivery, in some cases, is known to prevent or delay skin-to-skin contact, postpone the first 
$\mathrm{BF}$, reduce the possibility of exclusive $\mathrm{BF}$ and increase the likelihood of formula supplementation [37-39]. Our data, however, showed that among women with CHTN who intended to $\mathrm{BF}$, the impact of a delay in early $\mathrm{BF}$ associated with cesarean delivery could be overcome with healthcare provider's commitment to $\mathrm{BF}$, multidisciplinary education and support of patients and families.

Limitations to this investigation are those inherent to the retrospective design and the lack of follow-up information regarding infant feeding after discharge. We also recognize that a uniform threshold for initiating antihypertensive therapy can only be established with a treatment trial. Also, the definition of BF initiation at discharge may be applicable only to women with high risk obstetrical conditions for whom early mother-infant contact may be delayed. The strength of this investigation rests on the size of the obstetrical and neonatal population and the fact that the data were obtained directly from contemporaneous medical records, and not via post-delivery maternal questionnaires.

In conclusion, infants born to women with both CHTN-meds and CHTN without meds are at a higher risk for premature birth and concurrent morbidities that often require admission to the NICU leading to temporary mother-infant separation. Intention to BF predicts better $\mathrm{BF}$ initiation rates, however, exclusive $\mathrm{BF}$ at discharge in women with $\mathrm{CHTN}$ fell short of the expected direct BF goal, raising concerns about long term $B F$ duration. Due to severity of illness, treatment side effects and infants' morbidities, dyads affected by CHTN who are not ready for early direct $\mathrm{BF}$, can be helped by temporary alternatives such as EBM and donor human milk. Additionally, the high rate of partial BF during their hospital stay may identify women who are still striving toward exclusive $\mathrm{BF}$ at discharge and who, with specific support and guidance, might achieve their intended goal.

\section{References}

1. Vidaeff A, Espinoza J, Simhan H, Pettker CM (2019) ACOG practice bulletin No. 203: Chronic hypertension in pregnancy. Obstet Gynecol 133: e26-e50.

2. Webster K, Fishburn S, Maresh M, Findlay SC, Chappell LC, et al. (2019) Diagnosis and management of hypertension in pregnancy: Summary of updated NICE guidance. BMJ 366: 15119.

3. Battarbee AN, Sinkey RG, Harper LM, Oparil S, Tita ATN et al. (2020) Chronic hypertension in pregnancy. Am J Obstet Gynecol 222: 532-541.

4. Bateman BT, Bansil P, Hernandez-Diaz S, Mhyre JM, Callaghan WM, et al. (2012) Prevalence, trends, and outcomes of chronic hypertension: A nationwide sample of delivery admissions. Am J Obstet Gynecol 206: 134.e1-134.e8.

5. Admon LK, Winkelman TNA, Moniz MH, Davis MM, Heisler $\mathrm{M}$, et al. (2017) Disparities in chronic conditions among women hospitalized for delivery in the united states 20052014. Obstet Gynecol 130: 1319-1326.

6. Ananth CV, Duzyj CM, Yadava S, Schwebel M, Tita ATN, et al. (2019) Changes in the prevalence of chronic hyper- tension in pregnancy, United States, 1970-2010. Hypertension 74: 1089-1095.

7. Webster LM, Conti-Ramsden F, Seed PT, Webb AJ, Nelson-Piercy C, et al. (2017) impact of antihypertensive treatment on maternal and perinatal outcomes in pregnancy complicated by chronic hypertension: A systematic review and meta-analysis. J Am Heart Assoc 6: 005526.

8. Ankumah NAE, Sibai BM (2017) Chronic hypertension in pregnancy: Diagnosis, management, and outcomes. Clin Obstet Gynecol 60: 206-214.

9. Chahine KM, Sibai BM (2019) Chronic hypertension in pregnancy: New concepts for classification and management. Am J Perinatol 3: 161-168.

10. Brown MA, Magee LA, Kenny LC, Karumanchi SA, McCarthy FP, et al. (2018) The hypertensive disorders of pregnancy: ISSHP classification, diagnosis \& management recommendations for international practice. Pregnancy Hypertens 13: 291-310.

11. Ram M, Berger H, Geary M, McDonald SD, Murray-Davis $B$, et al. (2018) Timing of delivery in women with chronic hypertension. Obstet Gynecol 132: 669-677.

12. Anderson PO (2018) Treating hypertension during breastfeeding. Breastfeed Med 13: 95-96.

13. Steube AM, Schwarz EB, Grewen K, Rich-Edwards JW, Michels KB, et al. (2011) Duration of lactation and incidence of maternal hypertension: A longitudinal cohort study. Am J Epidemiol 174: 1147-1158.

14. Gunderson EP, Hurston SR, Ning X, Lo JC, Crites $Y$, et al. (2015) Lactation and progression to type 2 diabetes mellitus: A prospective cohort study. Ann Intern Med 163: 889-898.

15. Nguyen B, Jin K, Ding D (2017) Breastfeeding and maternal cardiovascular risk factors and outcomes: A systematic review. PLoS One 12: e0187923.

16. Ramji N, Challa S, Murphy PA, Quinlan J, Crane JMG, et al. (2018) A comparison of breastfeeding rates by obesity class. J Matern Fetal Neonatal Med 31: 3021-3026.

17. Flegal KM, Kruszon-Moran D, Carroll MD, Fryar CD, Ogden C (2016) Trends in obesity among adults in the united states, 2005 to 2014. JAMA 315: 2284-2291.

18. Bramham K, Parnell B, Nelson-Piercy C, Seed PT, Poston L, et al. (2014) Chronic hypertension and pregnancy outcomes: Systematic review and meta-analysis. BMJ 348: 2301.

19. Moussa HN, Leon MG, Marti A, Chediak A, Pedrosa C, Blackwell SC, et al. (2017) Pregnancy outcomes in women with preeclampsia superimposed on chronic hypertension with and without severe features. Am J Perinatol 34: 403408.

20. Cordero L,Valentine CJ, Samuels P, Giannone PJ, Nankervis CA (2012) Breastfeeding in women with severe preeclampsia. Breastfeed Med 7: 457-463.

21. Cordero L, Bajwa A, Prasad MR, Oza-Frank R, Landon MB, et al. (2016) Breastfeeding at hospital discharge among high risk obstetrical patients. Int $\mathrm{J}$ Womens Health Wellness 2: 031.

22. Binns C, Lee M, Low WY (2016) The long-term public health benefits of breastfeeding. Asia Pac J Public Health 28: 7-14.

23. Fox R, Kitt J, Leeson P, Aye CYL, Lewandowski AJ (2019) Preeclampsia: Risk factors, diagnosis, management, and the cardiovascular impact on the offspring. J Clin Med 8: 1625. 
24. Cordero L, Thung S, Landon MB, Nankervis CA (2014) Breastfeeding initiation failure in women with pregestational diabetes mellitus. Clin Pediatr 53: 18-25.

25. Centers for Disease Control and Prevention (2016) Breastfeeding report card: United States 2018, Atlanta, GA: Centers for disease control and prevention.

26. Dolin C, Kominiarek MA (2018) Pregnancy in women with obesity. Obstet Gynecol Clin N Am 45: 217-232.

27. Feldman-Winter L, Douglass-Bright A, Bartick MC, Matranga J (2013) The new mandate from the joint commission on the perinatal care core measures of exclusive breastmilk feeding: Implications for practice and implementation in the united states. J Hum Lact 29: 291-295.

28. Wight N, Marinelli KA, Academy of breastfeeding medicine (2014) ABM clinical protocol \#1: Guidelines for blood glucose monitoring and treatment of hypoglycemia in term and late-preterm neonates, revised 2014. Breastfeed Med 9: 173-179.

29. Boies EG, Vaucher YE (2016) ABM clinical protocol \#10: breastfeeding the late preterm (34-36 6/7 weeks of gestation) and early term infants (37-38 6/7 weeks of gestation), second revision 2016. Breastfeed Med 11: 494-500.

30. Kellams A, Harrel C, Omage S, Gregory C, Rosen-Carole C (2017) ABM clinical protocol \#3: supplementary feedings in the healthy term breastfed neonate, revised 2017 . Breastfeed Med 12: 188-198.

31. Forster DA, Johns HM, McLachlan HL, Moorhead AM, McEgan KM, et al. (2015) Feeding infants directly at the breast during the postpartum hospital stay is associated with increased breastfeeding at 6 months postpartum: A prospective cohort study. BMJ Open 5: 007512.
32. Felice JP, Cassano PA, Rasmussen KM (2016) Pumping human milk in the early postpartum period: Its impact on long-term practices for feeding at the breast and exclusively feeding human milk in a longitudinal survey cohort. Am J Clin Nutr 103: 1267-1277.

33. Pinchevski-Kadir S, Shust-Barequet S, Zajicek M, Leibovich M, Strauss T, et al. (2017) Direct feeding at the breast is associated with breast milk feeding duration among preterm infants. Nutrients 9: 1210.

34. Bai DL, Fong DYT, Lok KYW, Wong JYH, Tarrant M (2016) Practices, predictors and consequences of expressed breast-milk feeding in healthy full-term infants. Public Health Nutr 20: 492-503.

35. Keim SA, Boone KM, Oza-Frank R, Geraghty SR (2017) Pumping milk without ever feeding at the breast in the moms2moms study. Breastfeed Med 12: 422-429.

36. Cordero L, Oza-Frank R, Landon MB, Nankervis CA (2015) Breastfeeding initiation among macrosomic infants born to obese nondiabetic mothers. Breastfeed Med 10: 239-245.

37. Moore ER, Bergman N, Anderson GC, Medley N (2016) Early skin-to-skin contact for mothers and their healthy newborn infants. Cocrane Database Syst Rev 11: CD003519.

38. Hobbs AJ, Mannion CA, McDonald SW, Brockway M, Tough SC (2016) The impact of caesarean section on breastfeeding initiation, duration and difficulties in the first four months postpartum. BMC Pregnancy Childbirth 16: 90.

39. Walweiner S, Müller M, Doster A, Plewniok K, Christian W, et al. (2016) Predictors of impaired breastfeeding initiation and maintenance in a diverse sample: What is important? Arch Gynecol Obstet 294: 455-466. 